Annales Academiæ Scientiarum Fennicæ

Series A. I. Mathematica

Volumen 2, 1976, 203-232
Commentationes in honorem

Rolf Nevanlinna

LXXX annos nato

\title{
CARATHÉODORY BODIES
}

MAURICE HEINS

1. Introduction. This paper treats problems of Pick-Nevanlinna type for positive harmonic functions via an extension of Carathéodory's original approach to the theory of coefficient bodies [3]. We consider a non-compact Riemann surface $F$ having finite topological characteristics none of whose boundary components is pointlike. We fix a point $a \in F$ and denote by $P$ the family of positive harmonic functions on $F$ which take the value 1 at $a$. We denote by $A$ a continuous linear map of the space of realvalued harmonic functions on $F$ (endowed with the topology of uniform convergence on compact subsets of $F$ ) into a fixed Banach space over the real field. By the Carathéodory body associated with $P$ and $\Lambda$ we understand $\Lambda(P)$, i.e., the image of $P$ with respect to $\Lambda$. In this paper, we shall be concerned with the special map $\Lambda$ given explicitly in $\$ 2$. Here the fixed Banach space is a real finite dimensional number space. An important feature of the so chosen $A$ is the fact that the problem of characterizing $\Lambda(P)$ and determining the preimages of its points with respect to $\Lambda \mid P$ subsumes the Pick-Nevanlinna problem for $F$. The reason that $\Lambda(P)$ is accessible to study appears on introducing a Schottky double $S$ of $F$. For then $\Lambda(P)$ may be shown to be representable as the convex hull of the image of fr $F$ with respect to a map defined in terms of meromorphic functions on $S$ about which functions a fair amount of information is available. We are referring to the map $\Phi$ of $\S 3$ which is the surrogate in our study of Carathéodory's spherical norm curve [3]. It is a very special feature of $\Phi$ that its components are given in terms of abelian differentials on $S$ which are specified by $A$. Thus the apparatus of the theory of abelian differentials on compact Riemann surfaces is made available. When $F$ is the open unit disk, $\Phi$ may be given explicitly in terms of rational functions. Cf. (3.14), (3.15).

The map $\Phi$ will permit us to obtain a quantitative treatment for the Pick-Nevanlinna problem for $F$ in terms of Minkowski support functions (§6) and thereupon - as a byproduct - a solution of the Painlevé problem. To be sure, the results have a theoretical character. 
A central property of $\Lambda(P)$ for the $\Lambda$ under consideration is the fact (Theorem 4.1) that the determinacy subset of $\Lambda(P)$, i.e., the set of points of $\Lambda(P)$ having exactly one preimage in $P$ with respect to $\Lambda$, is exactly fr $\Lambda(P)$. The result is well-known in the classical situation of the Carathéodory theory. Cf. [3], § 12. The proof of Theorem 4.1 will be based on a "mixed method" allowing us to operate with analytic functions on $F$ which take values of modulus less than one and to which the lemma of Julia-Carathéodory-Wolff may be conveniently applied.

Thanks to the information which is available concerning the number of poles of the components of $\Phi$, the upper bound given by Carathéodory for the number of points of a spherical norm curve lying on a given supporting plane of its convex hull $([3], \S 11)$ may be generalized for the case of $\Phi$ and a supporting plane of $\Lambda(P)$. The extended bound, namely (3.19), is given in terms of indices entering in the definition of $\Lambda$. It will be seen that the preimage of a point of determinacy with respect to $\Lambda \mid P$ is the sum of a finite number of minimal positive harmonic functions on $F$, the number not exceeding the bound given by (3.19). The bounds given for the valence of extremals (in the sense of $R$. Nevanlinna [10]) for a finite Pick-Nevanlinna interpolation problem on a plane multiply-connected region of finite connectivity by Garabedian [5], for the Schwarz Lemma situation on a surface $F$ by Ahlfors [1], and for a finite Pick-Nevanlinna interpolation problem on $F$ by the author [6] are all consequences of this result. We remark that the problem of determining the extremal functions for the Schwarz Lemma situation studied by Ahlfors (loc.cit.) may be treated in terms of the support function of $\Lambda(P)$ which, in turn, is given in terms of $\Phi$.

2. The map $\Lambda$. We fix a Schottky double, $S$, of $F$. The components of $\Gamma=$ fr $F$ are regular analytic closed Jordan curves. We let $c$ denote their number. We let $g$ denote the genus of $F$. We let $\alpha$ denote the anticonformal map of $S$ onto itself keeping each point of $\Gamma$ fixed. It maps $F$ onto $S-\bar{F}$.

The period components of $A$. We fix $2 g+(c-1)$ regular analytic closed Jordan curves in $F$, say $\gamma_{1}, \cdots, \gamma_{2 g+(c-1)}$, which have the property that the homology classes of which they are members form a basis for the 1-dimensional homology group of $F$. We introduce

$$
\omega\left(u ; \gamma_{k}\right)=-i \int_{\gamma_{k}} \delta u, \quad k=1, \cdots, 2 g+(c-1),
$$

where $u$ is harmonic on $F$ and $\delta u$ denotes the abelian differential given 
in terms of a local uniformizer $\theta$ by $2(u \circ \theta)_{z} d z$. Of course $\omega\left(u ; \gamma_{k}\right)$ is simply the period of the conjugate of $u$ along $\gamma_{k}$.

The interpolation functionals. Let $B$ denote a finite set of points of $F$ including $a$. For each $b \in B-\{a\}$ we fix a regular analytic Jordan arc $x_{b}(\subset F)$ with initial point $a$ and terminal point $b$. With each $b \in B$ we associate a uniformizer $\theta_{b}$ satisfying $\theta_{b}(0)=b$ and a nonnegative integer $n(b)$. For $b \in B-\{a\}$ we define $l(u ; b, 0)$ by

$$
l(u ; b, 0)=\int_{\psi_{b}} \delta u,
$$

and for arbitrary $b \in B$ we define $l(u ; b, k), 1 \leqq k \leqq n(b)$, by

$$
l(u ; b, k)=2\left(u \circ \theta_{b}\right)_{z}^{(k-1)}(0) / k !,
$$

that is, as the $k$ th Taylor coefficient at 0 of a function analytic at 0 with real part given locally by $u \circ \theta_{b}$.

We now define $\Lambda(u)$ in the following way. We introduce

$$
\begin{aligned}
m= & 2 g+(c-1)+2 n(a)+2 \sum_{b \in B-\{a\}}[n(b)+1] \\
& =\chi-1+2 \sum_{b \in B}[n(b)+1]
\end{aligned}
$$

$\chi$ being the Euler characteristic of $\bar{F}$. We suppose that we are concerned with the nontrivial situation where $m>0$. We let $\sigma$ denote a $(1,1)$-map of $\{1, \cdots, m\}$ onto the set whose elements consist of: (I) the $\gamma_{k}$, (II) the ordered triples $(a, 1, k),(a, 2, k), k=1, \cdots, n(a)$, (III) the ordered triples $(b, 1, k),(b, 2, k), k=0, \cdots, n(b), b \in B-\{a\}$. For convenience we suppose that $\sigma$ maps $\{1, \cdots, \chi+1\}$ onto $\left\{\gamma_{1}, \cdots, \gamma_{\chi+1}\right\}$. We define $\Lambda(u)$ as the element of $R^{m}$ whose $j$ th component is $\omega[u ; \sigma(j)]$ when $\sigma(j)$ is one of the $\gamma_{k}$, is $\operatorname{Re} l(u ; b, k)$ when $\sigma(j)=(b, 1, k)$ and is $\operatorname{Im} l(u ; b, k)$ when $\sigma(j)=(b, 2, k)$.

[It is to be remarked that other formulations could be made for a map $\Lambda$. For example, one could consider $\Lambda$ as a map whose image lies in the set of real-valued functions with domain the set of elements specified by (I), (II), (III). For our purposes the question is not an important one.]

It is routine to verify that $\Lambda$ is a continuous linear map of the space of real harmonic functions on $F$ into $R^{m}$ in the sense indicated in $\S 1$. Clearly $\Lambda(P)$ is convex. That it is compact follows from the compactness properties of $P$.

3. The map $\Phi$. Our aim is to exhibit a map $\Phi$ from $S$ into $\hat{C}^{m}$, each component of which is meromorphic on $S$ and takes finite real values on $\Gamma$, such that $\Lambda(P)$ is the convex hull of $\Phi(T)$. Here $\hat{C}$ denotes the 
extended complex plane. To that end, we introduce for each $q \in \Gamma$ the minimal positive harmonic function on $F$ vanishing continuously at each point of $\Gamma$ save $q$ and normalized to take the value 1 at $a$. We denote it by $u_{q}$. We shall show that there exists an admitted $\Phi$ having the property that

$$
\Phi(q)=\Lambda\left(u_{q}\right), \quad q \in \Gamma .
$$

By the identity theorem there is at most one $\Phi$ satisfying (3.1). Thanks to the Riesz-Herglotz representation for positive harmonic functions on $F$ in terms of the $u_{q}$ (cf. [7], 93-101) we see that $\Lambda(P)$ is the convex hull of $\left\{\Lambda\left(u_{q}\right): q \in \Gamma\right\}$, that is, $\Lambda(P)$ is the convex hull of $\Phi(\Gamma)$. We continue describing the construction of $\Phi$ and studying some of its important properties.

As a first step we establish meromorphic prolongation to $S$ of the functions

$$
\begin{aligned}
& q \rightarrow \omega\left(u_{q} ; \gamma_{j}\right), \quad j=1, \cdots, \chi+1 \\
& q \rightarrow l\left(u_{q} ; b, 0\right), \quad b \in B-\{a\}
\end{aligned}
$$

and

$$
q \rightarrow l\left(u_{q} ; b, k\right), \quad b \in B, 1 \leqq k \leqq n(b) .
$$

Once we show that this is the case, we see that the same is true for the real and imaginary components of the functions (3.3) and (3.4). Indeed, if $f$ is meromorphic on $S$ and takes finite values on $\Gamma$, then $(f+\overline{f \circ \alpha}) / 2$ is meromorphic on $S$ and is equal to $\operatorname{Re} f$ on $\Gamma$ and the corresponding observation holds relative to $(f-\overline{f \circ \alpha}) /(2 i)$ and $\operatorname{Im} f$. The existence of $\Phi$ with the stated properties is thereupon assured.

We start with the question of the meromorphic prolongation of the functions (3.2). We introduce $G_{s}$, Green's function for $F$ with pole $s$ and recall the classical result

$$
u_{q}=\lim _{s \rightarrow q} G_{s} / G_{s}(a) .
$$

Now (2.1) is defined for the functions $G_{s} / G_{s}(a)$ with $s \notin \gamma_{j}$ and we have

$$
\begin{aligned}
\omega\left(u_{q} ; \gamma_{j}\right) & =-i \lim _{s \rightarrow q}\left[G_{s}(a)\right]^{-1} \int_{\gamma_{j}} \delta G_{s} \\
& =-i \lim _{s \rightarrow q}\left[G_{a}(s)\right]^{-1} \int_{\gamma_{j}} \delta G_{s},
\end{aligned}
$$

making use of the symmetry of Green's function. The function 


$$
s \rightarrow-i \int_{\gamma_{j}} \delta G_{s}
$$

is harmonic on $F-\gamma_{j}$ and vanishes continuously on $\Gamma$. It admits a harmonic prolongation $H_{j}$ to $S-\left\{\gamma_{j}, \alpha\left(\gamma_{j}\right)\right\}$ by means of Schwarzian reflexion across $\Gamma$. It is to be observed that $H_{j}$ admits harmonic prolongation across $\gamma_{j}$ (resp. $\left.\alpha\left(\gamma_{j}\right)\right)$ from each side and that the prolongations in question differ from one another in some neighborhood of $\gamma_{j}$ (resp. $\alpha\left(\gamma_{j}\right)$ ) by $2 \pi$. It follows from this observation that $\delta H_{j}$ is not the zero differential and that $\delta H_{j}$ admits prolongation to an abelian differential of the first kind on $S$. We make the harmless convention of denoting the prolongation of $\delta H_{j}$ to $S$ also by $\delta H_{j}$. Using (3.6) we conclude that (3.2) is the restriction to $\Gamma$ of $\delta H_{j} / \delta G_{a}$, with $\delta G_{a}$ construed as the differential of the prolongation to $S$ by Schwarzian reflexion of Green's function for $F$ with pole $a$. This convention will prevail throughout. The divisor of each $\delta H_{j} / \delta G_{a}$ is at least as large as $-\partial \delta G_{a}$. The notation $\partial_{\omega}$ (resp. $\left.\partial_{f}\right)$ is used to denote the divisor of an abelian differential $\omega$ (resp. a meromorphic function $f$ ) on $S$. It is to be observed that the functions $\delta H_{j} / \delta G_{a}$ are determined by the homology classes of the $\gamma_{j}$ (in the sense of $F$ ).

The remaining functions, (3.3) and (3.4), may be treated similarly. They will be seen to be restrictions to $\Gamma$ of quotients of abelian differentials on $S$, the denominator in every case being $\delta G_{a}$. For the functions (3.3) the numerator is an abelian differential of the third kind having poles at $a$ and $b$. For the functions (3.4) the numerator is an abelian differential of the second kind having a pole of order $k+1$ at $b$.

The functions (3.3). We proceed taking into account the definition of $l\left(u_{q} ; b, 0\right)$ and the representation (3.5) of $u_{q}$. We observe that the function defined on $F-\varkappa_{b}$ by

$$
s \rightarrow \int_{x_{b}} \delta G_{s}
$$

is harmonic and vanishes continuously on $\Gamma$, and consequently, admits harmonic prolongation to $S-\left\{x_{b}, \alpha\left(x_{b}\right)\right\}$ by Schwarzian reflexion across $\Gamma$. The prolongation in question will be denoted by $K_{b}$. On introducing a local uniformizer $\theta$, satisfying $\theta(0)=a$, and using standard properties of Green's function and (3.8) we obtain the local relation

$$
K_{b}[\theta(z)]=\lambda(z)+h(z),
$$

where $\lambda$ is an analytic logarithm of the identity map on a neighborhood of 0 from which a slit terminating at 0 has been removed and $h$ is harmonic on a neighborhood of 0 . A corresponding remark holds con- 
cerning the local behavior of $K_{b}$ near $b$ with $\lambda$ this time being the negative of an analytic logarithm of the identity map on a slit neighborhood of 0 . By Schwarzian reflexion one obtains similar results for the behavior of $K_{b}$ near $\alpha(a)$ and $\alpha(b)$, save that the $\lambda$ term appears in the guise of the conjugate of an analytic function.

We note further that $K_{b}$ admits harmonic prolongation across $x_{b}-\{a, b\}$ from each side and that the prolongations in question differ from one another by $2 \pi i$ in some neighborhood of each point of $\varkappa_{b}-\{a, b\}$. Corresponding observations hold relative to the prolongation of $K_{b}$ across $\alpha\left(\varkappa_{b}-\{a, b\}\right)$.

It now follows that $\delta K_{b}$ admits prolongation to an abelian differential on $S$ which will be denoted also by $\delta K_{b}$. The latter is an abelian differential of the third kind with poles at $a$ and $b$, the residue of which is 2 at $a$ and is -2 at $b$. This observation is a consequence of the stated properties of $K_{b}$. It is to be noted that the operator $\delta$ has the effect of annihilating the conjugate analytic terms entering in the local representation of $K_{b}$ near $\alpha(a)$, resp. $\alpha(b)$.

The remainder of the argument follows that given in the case of (3.2). Indeed,

$$
l\left(u_{q} ; b, 0\right)=\lim _{s \rightarrow q}\left[G_{a}(s)\right]^{-1} \int_{\varkappa_{b}} \delta G_{s}, \quad s \in F .
$$

It follows that (3.3) is the restriction to $\Gamma$ of $\delta K_{b} / \delta G_{a}$.

The functions (3.4). We recall that the functional (2.3) is specified in terms of a local uniformizer $\theta_{b}, \theta_{b}(0)=b$, and an index $k, 1 \leqq k \leqq n(b)$. We first consider the Taylor coefficients at 0 of twice the complex differential coefficient $h_{z}$ where

$$
h(z)=G_{s}\left[\theta_{b}(z)\right]
$$

and their dependence on the parameter $s$. An examination of these coefficients shows that

$$
L_{b, k}: s \rightarrow l\left(G_{s} ; b, k\right)
$$

is a complex-valued harmonic function on $F-\{b\}$ which vanishes continuously on $\Gamma$ and is such that for $z$ small we have

$$
L_{b, k}\left[\theta_{b}(z)\right]=\frac{z^{-k}}{k}+<\cdots>,
$$

where the omitted term is harmonic at 0 . The prolongation of $L_{b, k}$ to $S-\{b, \alpha(b)\}$ by Schwarzian reflexion will be denoted also by $L_{b, k}$. The operator $\delta$ induces from the latter function an abelian differential on 
$S$ of the second kind (denoted $\delta L_{b, k}$ ) having a pole of order $k+1$ at $b$. It is to be observed that, as above, the operator $\delta$ annihilates the singularity of $L_{b, k}$ at $\alpha(b)$ because of its conjugate analytic character. From

$$
l\left(u_{q} ; b, k\right)=\lim _{s \rightarrow q} \frac{L_{b, k}(s)}{G_{a}(s)}, \quad s \in F,
$$

we conclude that (3.4) is the restriction to $\Gamma$ of $\delta L_{b, k} / \delta G_{a}$.

The case of the unit disk. Here it is a simple matter to determine $\delta K_{b} / \delta G_{a}$ and $\delta L_{b, k} / \delta G_{a}$ with $\theta_{b}(z)=z+b$. Because of the simple connectivity of the unit disk the $\delta H_{j} / \delta G_{\alpha}$ do not come into consideration. We have:

$$
\begin{aligned}
\delta G_{a} & =-\frac{1-|a|^{2}}{(z-a)(1-\bar{a} z)} d z, \\
\delta K_{b} & =2\left[(z-a)^{-1}-(z-b)^{-1}\right] d z, \\
\delta L_{b, k} & =-2(z-b)^{-(k+1)} d z,
\end{aligned}
$$

and consequently we conclude

$$
\frac{\delta K_{b}}{\delta G_{a}}=\frac{2(b-a)(1-\bar{a} z)}{(z-b)\left(1-|a|^{2}\right)}
$$

and

$$
\frac{\delta L_{b, k}}{\delta G_{a}}=\frac{2(z-a)(1-\bar{a} z)}{(z-b)^{k+1}\left(1-|a|^{2}\right)} .
$$

With $a=b=0,(3.15)$ yields essentially the factor occurring in Carathéodory's classical theory.

We continue our study of $\Phi$ and seek an upper estimate for the number of poles counted by multiplicity of a linear combination $f$ with constant coefficients of the component functions entering in the definition of $\Phi$. The only points of $S$ at which these functions may have poles are the points of $B \cup \alpha(B)$ and the zeros of $\delta G_{a}$. On examining the functions $\delta H_{j} / \delta G_{a}, \delta K_{b} / \delta G_{a}, \delta L_{b, k} / \delta G_{a}$ and the functions obtained from the functions $\delta K_{b} / \delta G_{a}$ and $\delta L_{b, k} / \delta G_{a}$ by composition with $\alpha$ and conjugation we obtain the following lower estimate for $\partial_{f}$ :

$$
\left\{\begin{array}{lll}
\partial_{f}(a), & \partial_{f}[\alpha(a)] \geqq-n(a) ; & \\
\partial_{f}(b), \partial_{f}[\alpha(b)] \geqq-n(b)-1-\partial_{\delta G_{a}}(b), & b \in B-\{a\} ; \\
\partial_{f}(c), \partial_{f}[\alpha(c)] \geqq-\partial_{\delta G_{a}}(c), & c \in \bar{F}-B .
\end{array}\right.
$$

It follows from (3.16) that 


$$
\sum_{p \in S} \min \left\{\partial_{f}(p), 0\right\} \geqq-2\left\{(\chi+1)+n(a)+\sum_{b \in B-\{a\}}[n(b)+1]\right\} .
$$

Hence the number of poles of $f$ counted by multiplicity does not exceed

$$
2\left\{\chi+\sum_{b \in B}[n(b)+1]\right\}=m+(\chi+1),
$$

where $m$, we recall, is given by (2.4). We note that the bound (3.18) does not exceed $2 m$ since $m \geqq \chi+1$. It will be seen that the bound (3.18) will play a role in the study of the members of $P$ which are preimages with respect to $\Lambda$ of points of determinacy of $\Lambda(P)$. In the classical case of the unit disk $\chi=-1$ and the bound reduces to $m$.

We continue our study of $\Phi$ and show

Theorem 3.1. $\Phi(T)$ does not lie in a $(m-1)$-dimensional hyperplane of $R^{m}$.

Before turning to the proof we make the following observations. The theorem is equivalent to the assertion that the differentials of the functions entering in the definition of $\Phi$ are linearly independent over $R$. The theorem taken in conjunction with the estimate (3.18) shows that a supporting hyperplane of $\Phi(T)$ has at most

$$
\chi+\sum_{b \in B}[n(b)+1] \quad(\leqq m)
$$

distinct points in common with $\Phi(\Gamma)$. In the classical case of Carathéodory where $F$ is the unit disk and $B=\{0\}$, we have $m=2 n(0)$ and the proof of Theorem 3.1 reduces to noting that a rational function, not identically zero, the sum of the orders of the poles of which does not exceed $2 n(0)$ has at most $2 n(0)$ zeros.

Proof of Theorem 3.1. Let $O=(0, \cdots, 0) \in R^{m}$. We note that $O$ is the image of the constant 1 with respect to $A$. Suppose that $\Phi(T)$ is contained in a $(m-1)$-dimensional hyperplane of $R^{m}$. Then $\Lambda(P)$, being the convex hull of $\Phi(T)$, is contained in the same hyperplane and, consequently, $O$ is a point of the hyperplane. It follows that the component functions of $\Phi$ are linearly dependent over $R$.

Let $\varphi$ denote a linear combination of the components of $\Phi$ with real coefficients which is identically zero. If not all the coefficients of the components arising from the $\delta L_{b, k} / \delta G_{a}$ (by the indicated process of forming $(f+\overline{f \circ \alpha)} / 2$ and $(f-\overline{f \circ \alpha}) /(2 i)$ from such a function $f)$ were zero, then $\varphi$ would have a pole at some $b$. This is not possible. Thereupon we consider the terms arising from the $\delta K_{b} / \delta G_{a}$ and conclude that if not all the coefficients of such terms were zero, $\varphi$ would have a pole at some $b$. Again, this is not possible. Thus $\varphi$ reduces to a linear combination of the $\delta H_{k} / \delta G_{a}$ with real coefficients. 
If $v$ is harmonic on a region of $S$ containing $\bar{F}$, then

$$
\omega\left(v ; \gamma_{k}\right)=\frac{i}{2 \pi} \int_{\Gamma}^{i} v \delta H_{k} .
$$

Further, there exist $\chi+1$ such $v$, say $v_{1}, \cdots, v_{\chi+1}$, such that

$$
\omega\left(v_{j} ; \gamma_{k}\right)=\delta_{j k}, \quad j, k=1, \cdots, \chi+1 .
$$

However, if

$$
\sum_{k} c_{k} \frac{\delta H_{k}}{\delta G_{a}}=0
$$

then $c_{k}$ being real, we have

$$
\sum_{k} c_{k} \omega\left(v_{j} ; \gamma_{k}\right)=0, \quad j=1, \cdots, \chi+1,
$$

and hence $c_{1}=\cdots=c_{\chi+1}=0$. We conclude that the components of $\Phi$ are linearly independent over $R$.

Theorem 3.1 now follows from the conclusions of the preceding two paragraphs. The statement of Theorem 3.1 may be strengthened to the assertion that for each component $\Gamma_{k}$ of $\Gamma, \Phi\left(\Gamma_{k}\right)$ does not lie in a $(m-1)$-dimensional hyperplane of $R^{m}$ as we see by appeal to the Identity Theorem.

It is now easy to conclude

Theorem 3.2. $O \in \operatorname{int} \Lambda(P)$.

Proof. If $O \notin \operatorname{int} \Lambda(P)$, then a supporting plane of $\Lambda(P)$ would pass through $O$. By Theorem 3.1 only a finite number of points of $\Phi(\Gamma)$ would lie in the supporting plane. It then follows that a preimage of $O$ with respect to $\Lambda$ in $P$ would be a finite sum of minimal positive harmonic functions on $F$. To see this, let $l$ be a linear homogeneous function on $C^{m}$ with real coefficients such that $\left\{x \in R^{m}: l(x)=0\right\}$ is the supporting plane in question with $\max _{\Lambda(P)} l=0$. With $\mu$ denoting the generating measure in the Riesz-Herglotz representation of a preimage $v$ of $O$ in $P$ with respect to $\Lambda$, we have

$$
\int_{\Gamma} l \circ \Phi(q) d \mu(q)=l\left[\int_{\Gamma} \Phi(q) d \mu(q)\right]=l[\Lambda(v)]=0 .
$$

Consequently, $\mu$ is comprised of a finite number of point masses, the number being at most $\chi+\sum_{b \in B}[n(b)+1]$ by (3.18) since each $q$ for which $\Phi(q)$ is in the supporting plane is a zero of even multiplicity of $l \circ \Phi$. Hence $v$ is the sum of a positive number of minimal positive harmonic functions on $F$ and consequently is not bounded above. How- 
ever, the constant 1 is a preimage of $O$ in $P$ with respect to $A$. Contradiction. Theorem 3.2 follows.

We remark that one may also proceed in the inverse direction. That is, Theorem 3.2 may be proved, without appeal to Theorem 3.1, by exhibiting a subfamily of $P$ whose image with respect to $\Lambda$ is a neighborhood of $O$. Theorem 3.1 may then be concluded on the basis of the fact that $\Lambda(P)$ is the convex hull of $\Phi(T)$.

4. The determinacy subset of $\Lambda(P)$. The notion of the determinacy subset of $\Lambda(P)$ has already been introduced in $\S 1$. We let $D$ denote this set. We show

Theorem 4.1. $D=$ fr $\Lambda(P)$.

Proof. We first show

$$
\operatorname{int} \Lambda(P) \subset \Lambda(P)-D,
$$

from which it follows that

$$
D \subset \text { fr } \Lambda(P) \text {. }
$$

Now $O$ is in the convex hull of $\Phi(T)$ and so $O$ admits a barycentric representation of the form $\sum_{1}^{m+1} \mu_{k} \Phi\left(q_{k}\right), q_{k} \in \Gamma, \mu_{k} \geqq 0, \sum \mu_{k}=1$. Hence $O$ is the image with respect to $A$ of both the constant 1 , which we shall denote by $w_{1}$, and $w_{2}=\sum \mu_{k} u_{q_{k}}$. Since $w_{1} \neq w_{2}, w_{2}$ being not bounded above, we conclude that $O \notin D$.

Given $x \in \operatorname{int} \Lambda(P), x \neq O$, we let $y$ denote the point of $\operatorname{fr} \Lambda(P)$ lying on the ray with initial point $O$ which passes through $x$, and let $t$ denote the positive number less than one such that $x=t y$. Let $v \in P$ be a preimage of $y$ with respect to $\Lambda$. Then

$$
(1-t) w_{k}+t v, \quad k=1,2,
$$

are distinct preimages of $x$ with respect to $\Lambda$ which are in $P$. Hence $x \notin D$. The inclusion (4.1) is thereby established and hence (4.2).

It remains to show

$$
\text { fr } \Lambda(P) \subset D
$$

For this part of the proof we shall make essential use of the barycentric representation method of the preceding paragraph as well as of the Lemma of Julia-Carathéodory-Wolff. Our procedure will depend upon a translation of the situation we are studying into one pertaining to analytic functions on $F$ taking values of modulus less than one.

Let $x \in \mathrm{fr} \Lambda(P)$. Then a preimage of $x$ with respect to $\Lambda$ lying in $P$ is the sum of a finite number of minimal positive harmonic functions on $F$. Cf. the proof of Theorem 3.2. Suppose that $U_{1}, U_{2} \in P$ satisfy 
$\Lambda\left(U_{1}\right)=\Lambda\left(U_{2}\right)=x$. We shall show that $U_{1}=U_{2}$. The inclusion (4.4) follows and consequently Theorem 4.1 is seen to hold.

We proceed to replace the $U_{k}$ by the real parts of analytic functions on $F$.

Case 1. $\chi \geqq 0$. Here we introduce a function $h$ harmonic on a region of $S$ containing $\bar{F}$ such that

$$
\omega\left(h ; \gamma_{k}\right)=-\omega\left(U_{1} ; \gamma_{k}\right), \quad k=1, \cdots, \chi+1,
$$

namely,

$$
h=A-\sum \omega\left(U_{1} ; \gamma_{k}\right) v_{k}
$$

where the $v_{k}$ are the functions introduced in $\S 3$ and $A$ is a large positive constant so chosen that $h \mid F>0$. Now the point

$$
\left(\omega\left(\frac{h}{h(a)} ; \gamma_{1}\right), \cdots, \omega\left(\frac{h}{h(a)} ; \gamma_{x+1}\right)\right)
$$

is in the convex hull of the image of $\Gamma$ with respect to

$$
q \rightarrow\left(\frac{\delta H_{1}}{\delta G_{a}}(q), \cdots, \frac{\delta H_{\chi+1}}{\delta G_{a}}(q)\right) .
$$

We conclude that there exists a positive harmonic function $H$, the sum of a finite number of minimal positive harmonic functions on $F$ satisfying

$$
\omega\left(H ; \gamma_{k}\right)=\omega\left(h ; \gamma_{k}\right), \quad k=1, \cdots, \chi+1 .
$$

It follows from (4.5) that $U_{j}+H, j=1,2$, is the real part of an analytic function on $F$.

Case 2. $\chi=-1$. Here $U_{j}, j=1,2$, is the real part of an analytic function on $F$ which is simply-connected in this case. We understand $H$ to be the constant zero.

We introduce $f_{j}$ analytic on $F$ satisfying: $\operatorname{Re} f_{j}=U_{j}+H$, $\operatorname{Im} f_{j}(a)=0, j=1,2$. We let $M$ denote the Möbius transformation

$$
z \rightarrow(z-1) /(z+1),
$$

and recall that $M$ maps $\{\operatorname{Re} z>0\}$ onto $\{|z|<1\}$. The ancillary function $v$ is defined by

$$
v=\operatorname{Re}\left[\operatorname{inv} M \circ\left(\frac{M \circ f_{1}+M \circ f_{2}}{2}\right)\right]-H .
$$

It is by studying $v$ that we shall see that $U_{1}=U_{2}$. It is clear that $v(a)=1$. We shall show in the following paragraphs that $v \in P$ and $\Lambda(v)=x$. Once these facts are established, using (4.11) and the fact that 
$v$ is the sum of a finite number of minimal positive harmonic functions on $F$, we are in a position to conclude that $U_{1}=U_{2}$ and thereupon that Theorem 4.2 holds. Indeed, on considering an open subare $\gamma$ of $\Gamma$ on which $v+H$ vanishes continuously, we see from (4.11) that $\left(M \circ f_{1}+M \circ f_{2}\right) / 2$ takes boundary values of modulus one on $\gamma$. Hence $M \circ f_{1}=M \circ f_{2}$ and so $U_{1}=U_{2}$ as we wished to show.

$v \in P$. The case where $\chi=-1$ is trivial and will be put aside. We note that in the remaining case

$$
H=\sum_{q \in E} \sigma(q) u_{q}
$$

where $E$ is a finite nonempty subset of $\Gamma$ and each $\sigma(q)$ is positive. The notation $u_{q}$ of $\S 3$ is to be recalled. Given $q \in E$, we introduce a univalent conformal map $\theta$ of $\{\operatorname{Re} z>0\}$ onto a small simply-connected Jordan region $\Omega$ in $F$ bounded by an open subarc of $T$ which contains $q$ and an arc lying in $F$ save for its endpoints which are to be the same as those of the subarc of $\Gamma$. We suppose that $\theta$ is normalized by the condition $\theta(\infty)=q, \theta$ being construed as continuously extended to the closure of $\{\operatorname{Re} z>0\}$ in the sense of $\hat{C}$. Suppose now that $w$ is a function analytic in $\Omega$ and taking values of modulus less than one. By the lemma of Julia-Carathéodory-Wolff inv $M \circ(w \circ \theta)$ admits a unique representation of the form

$$
A z+B(z)
$$

where $A$ is a nonnegative real number, $\operatorname{Re} B(z) \geqq 0$, and

$$
\lim z^{-1} B(z)=0,
$$

$z$ tending to $\infty$ sectorially. Further

$$
z[1-w \circ \theta(z)]
$$

possesses a limit as $z \rightarrow \infty$ sectorially, the value being $\infty$ when $A=0$ and otherwise $2 / A$. We apply this observation taking

$$
w=\left(M \circ f_{1}+M \circ f_{2}\right) / 2 .
$$

The sectorial limit of

$$
z\left[1-M \circ f_{j} \circ \theta(z)\right]
$$

as $z \rightarrow \infty$ is positive and finite. Indeed,

$$
\operatorname{Re} \operatorname{inv} M \circ\left(M \circ f_{j}\right) \circ \theta=\left(U_{j}+H\right) \circ \theta \geqq \sigma(q) u_{q} \circ \theta
$$

and so by the Lemma of Julia-Carathéodory-Wolff the coefficient $A$ of (4.13) for $w=M \circ f_{j}$ is at least as large as 


$$
\tau(q)=\inf _{\operatorname{Re} z>0}[\sigma(q) u \circ \theta(z) / \operatorname{Re} z],
$$

which is positive. Hence

$$
z\left\{1-\left[M \circ f_{1} \circ \theta(z)+M \circ f_{2} \circ \theta(z)\right] / 2\right\}
$$

possesses a sectorial limit as $z \rightarrow \infty$, not exceeding $2 / \tau(q)$. By the observation made relative to $(4.14)$, we see that

$$
\operatorname{Re}\left[\operatorname{inv} M \circ\left(\frac{M \circ f_{1}+M \circ f_{2}}{2}\right)\right] \circ \theta(z) \geqq \tau(q) \operatorname{Re} z .
$$

It follows by the boundary maximum principle for harmonic functions, (4.19), and the boundary behavior of $u_{q}$ that

$$
\operatorname{Re}\left[\operatorname{inv} M \circ\left(\frac{M \circ f_{1}+M \circ f_{2}}{2}\right)\right] \geqq \sigma(q) u_{q} .
$$

A second application of the boundary maximum principle taken with the boundary behavior of the $u_{q}$ shows that $v \geqq 0$. Thus we are assured that $v \in P$.

$\Lambda(v)=x$. We know at all events that

$$
\Lambda(v)=\Lambda\left[\operatorname{Re} \operatorname{inv} M \circ\left(\frac{M \circ f_{1}+M \circ f_{2}}{2}\right)\right]-\Lambda(H) .
$$

Hence the $k$ th component of $\Lambda(v)$ agrees with that of $x, k=$ $1, \cdots, \chi+1$, vacuously when $\chi=-1$.

Suppose that we are concerned with a component of $\Lambda(v)$ of the form $\operatorname{Re} l(v ; b, 0)$ or $\operatorname{Im} l(v ; b, 0)$. We have

$$
\begin{aligned}
l(v ; b, 0) & =\left[f_{1}(b)-f_{1}(a)\right]-l(H ; b, 0) \\
& =l\left(U_{1}+H ; b, 0\right)-l(H ; b, 0) \\
& =l\left(U_{1} ; b, 0\right) .
\end{aligned}
$$

This says that such a component of $\Lambda(v)$ agrees with the component of $x$ having the same index.

There remain to be studied the components deriving from functionals of the form $l(v ; b, k), k \geqq 1$. Essential use will be made of the fact that "order of contact" is preserved by composition with univalent analytic functions. The auxiliary map $M$ and its inverse enter in this connection. On introducing the local uniformizer $\theta$ used in the definition of $l(v ; b, k)$, we see that

$$
l(v ; b, k)=\frac{1}{k !}\left\{\left[\operatorname{inv} M \circ\left(\frac{M \circ f_{1}+M \circ f_{2}}{2}\right)\right] \circ \theta\right\}^{(k)}(0)-l(H ; b, k) .
$$


The Taylor coefficients at 0 of $f_{1} \circ \theta$ and $f_{2} \circ \theta$ agree for all indices not exceeding $k$. It follows by contact considerations that

$$
\operatorname{inv} M \circ\left(\frac{M \circ f_{1}+M \circ f_{2}}{2}\right) \circ \theta
$$

and $f_{1} \circ \theta$ have common Taylor coefficients at 0 for all indices not exceeding $k$. It suffices to use the elementary observation that if $f$ and $g$ are analytic at 0 and have common Taylor coefficients for all indices not exceeding $k(\geqq 0)$ and $\varphi$ is analytic at $f(0)=g(0)$, then $\varphi[g(z)]-$ $\varphi[f(z)]=O\left(|z|^{k+1}\right)$. Hence

$$
\begin{aligned}
l(v ; b, k) & =\frac{1}{k !}\left(f_{1} \circ \theta\right)^{(k)}(0)-l(H ; b, k) \\
& =l\left(U_{1}+h ; b, k\right)-l(H ; b, k) \\
& =l\left(U_{1} ; b, k\right) .
\end{aligned}
$$

It now follows that $\Lambda(v)=x$. The proof of Theorem 4.1 is thereby completed.

The following theorem is a consequence of Theorem 4.1 and the observation of the sentence following (3.23).

Theorem 4.2. If $u \in P$ satisfies $\Lambda(u) \in D$, then $u$ may be represented as the sum of at most

$$
\chi+\sum_{b \in B}[n(b)+1]
$$

minimal positive harmonic functions on $F$.

We shall see in $\S 5$ that the bound (4.26) cannot be ameliorated for assigned $\chi$ and $\sum[n(b)+1]$.

Theorem 4.1 permits us to obtain information concerning the points of $\Phi(\Gamma)$ lying in a given supporting plane of $\Lambda(P)$. We have

Theorem 4.3. Let $\Pi$ denote a supporting plane of $\Lambda(P)$ and let $q_{1}, \cdots, q_{n}$ be the distinct points of $\Gamma$ (supposed $n$ in number) such that $\Phi\left(q_{k}\right) \in \Pi$. Then $\Phi\left(q_{1}\right), \cdots, \Phi\left(q_{n}\right)$ are linearly independent (over $R$ ). Each $\Phi\left(q_{k}\right)$ is an extreme point of $\Lambda(P)$.

Proof. Let $\Pi$ be given by $l(y)=c(\neq 0)$, where $l$ is a linear function on $R^{m}$. If $\Phi\left(q_{1}\right), \cdots, \Phi\left(q_{n}\right)$ are linearly dependent so that $\sum t_{k} \Phi\left(q_{k}\right)=0$, where the $t_{k}$ are real and not all zero, then

$$
0=l\left[\sum t_{k} \Phi\left(q_{k}\right)\right]=\left(\sum t_{k}\right) c .
$$

Hence $\sum t_{k}=0$. From the fact that the sum of the positive $t_{k}$ plus the sum of the negative $t_{k}$ is zero, we conclude the existence of distinct members of $P$, say $U_{1}$ and $U_{2}$, with $\Lambda\left(U_{1}\right)=\Lambda\left(U_{2}\right) \in \Pi$. Contradiction. 
The fact that each $\Phi\left(q_{k}\right)$ is an extreme point of $\Lambda(P)$ may be seen as follows. If $\Phi\left(q_{k}\right)=(1-t) x_{1}+t x_{2}, 0<t<1, x_{1}, x_{2} \in \Lambda(P)$, then $x_{1}$ and $x_{2}$ belong to $\Pi$ and hence to $D$. With $U_{x_{j}} \in P$ satisfying $\Lambda\left(U_{x_{j}}\right)$ $=x_{j}, j=1,2$, we have $(1-t) U_{x_{1}}+t U_{x_{2}}=u_{q_{k}}$. Since $u_{q_{k}}$ is minimal, $U_{x_{1}}=U_{x_{2}}=u_{q_{k}}$. Hence $x_{1}=x_{2}$. Consequently, $\Phi\left(q_{k}\right)$ is an extreme point of $\Lambda(P)$.

It is obvious from Theorem 4.3 that $x \in \Lambda(P) \cap \Pi$, which admits a unique representation of the form $\sum_{1}^{n} t_{k} \Phi\left(q_{k}\right), t_{k} \geqq 0, \sum_{1}^{n} t_{k}=1$, admits a unique representation of the form $\sum_{1}^{n} t_{k} \Phi\left(q_{k}\right), t_{k}$ real. In principle, the $t_{k}$ may be calculated in terms of $x$ and the $\Phi\left(q_{k}\right)$ with the aid of determinants, the $\Phi\left(q_{k}\right)$ being linearly independent.

5. Some applications. (a) Augmented maps. Given $\Lambda$ we introduce a linear functional $l_{1}$ of the kind $u \rightarrow l(u ; b, k)$, where $k=0$ when $b \in F-B$ and $k=n(b)+1$ when $b \in B$, and we let $\Lambda_{1}$ denote the map of the space of real-valued harmonic functions on $F$ into $R^{m+2}$ for which the first $m$ components of $\Lambda_{1}(u)$ are the same as the respective components of $\Lambda(u)$, the component of index $m+1$ of $\Lambda_{1}(u)$ is $\operatorname{Re} l_{1}(u)$, and the component of index $m+2$ is $\operatorname{Im} l_{1}(u)$. We term $\Lambda_{1}$ an augmentation of $\Lambda$. Let $x$ be a given point of int $\Lambda(P)$. We associate with $x$ the set $V(x)$ given by

$$
V(x)=\left\{l_{1}(u): u \in P, \Lambda(u)=x\right\} .
$$

It is, of course, a nonempty compact convex subset of $C$. We show

Theorem 5.1. Given $u \in P$ such that $\Lambda(u)=x$. Then $\Lambda_{1}(u)$ is a point of determinacy of $\Lambda_{1}(P)$ if and only if $l_{1}(u) \in \operatorname{fr} V(x)$.

Corollary 5.2. int $V(x) \neq \varnothing$.

Proof of Theorem 5.1. We shall establish the theorem contrapositively. Suppose that $\Lambda_{1}(u)$ is not a point of determinacy of $\Lambda_{1}(P)$. Then $\Lambda_{1}(u) \in \operatorname{int} \Lambda_{1}(P)$. Then for some neighborhood $N$ of $l_{1}(u)$ it is the case that $\Lambda_{1}(P)$ contains all points of $R^{m+2}$ whose first $m$ components are the same as those of $x$ and whose last two components, $y_{m+1}, y_{m+2}$, are such that $y_{m+1}+i y_{m+2} \in N$. Hence $l_{1}(u) \notin$ fr $V(x)$.

Suppose now that $l_{1}(u) \in$ fr $V(x)$. Then $l_{1}(u) \in \operatorname{int} V(x)$. We note that for some $v \in P$, satisfying $\Lambda(v)=x$ the point $\Lambda_{1}(v)$ is not a point of determinacy of $\Lambda_{1}(P)$ since not every $v \in P$ satisfying $\Lambda(v)=x$ is a finite sum of minimal positive harmonic functions on $F$. Hence there exist distinct members of $P$, say $v_{1}$ and $v_{2}$, satisfying $\Lambda\left(v_{1}\right)=$ $\Lambda\left(v_{2}\right)=x$ and $\Lambda_{1}\left(v_{1}\right)=\Lambda_{1}\left(v_{2}\right)$. Further there exist $t, 0<t<1$, and $w \in P$ satisfying $\Lambda(w)=x$, such that

$$
l_{1}(u)=(1-t) l_{1}\left(v_{k}\right)+t l_{1}(w), \quad k=1,2 .
$$


We conclude that $\Lambda_{1}(u)$ is not a point of determinacy of $\Lambda_{1}(P)$ since $\Lambda_{1}(u)=\Lambda_{1}\left[(1-t) v_{k}+t w\right], k=1,2$, and the functions $(1-t) v_{k}+t w$ are distinct. Theorem 5.1 follows.

Proof of Corollary 5.2. If int $V(x)=\varnothing$, then for all $u \in P$ satisfying $\Lambda(u)=x, \Lambda_{1}(u)$ would be a point of determinacy of $\Lambda_{1}(P)$. Hence all such $u$ would be finite sums of minimal positive harmonic functions. This is not the case. The corollary follows.

Remark. It is clear that Theorem 5.1 and Corollary 5.2 admit extension to the situation where $\Lambda$ is "augmented" by several $l$.

(b) Garabedian's bound for the valence of an extremal solution of a PickNevantinna interpolation problem. As noted in § 1, P. Garabedian [5] gave a bound for the valence of the extremal functions associated with a finite Pick-Nevanlinna interpolation problem for a finitely connected plane region with nondegenerate boundary components where interpolation conditions of order zero are assigned at $n$ distinct points and there is more than one interpolating function. [The validity of the bound for interpolation conditions not necessarily of zero order was noted without proof.] He showed that the extremal interpolating functions corresponding to the frontier points of the Wertevorrat at a point different from the $n$ given points were maps of the region onto the open unit disk having a constant valence with value not exceeding $n+(\chi+1)=(n+1)+\chi$. [We shall recall the formal definitions of "Wertevorrat" and "extremal" below.] Ahlfors [1] obtained the corresponding bound for the "Schwarz Lemma" situation for a Riemann surface $F$ of the type considered in the present paper. Here $n=1$, there being one interpolation condition of zero order. Ahlfors' bound is $2+\chi$. The author [6] has extended the bound of Garabedian to the case of a finite Pick-Nevanlinna interpolation problem on a Riemann surface $F$, the orders of the interpolation conditions being not necessarily zero. To obtain the extended bound use was made of a variational formula of F. Riesz [13] applied to functions in a Hardy class, the theorem of Cauchy-Read [12], and a limiting argument. We shall now see that the extended Garabedian bound is a straightforward consequence of $(4.26)$.

The finite Pick-Nevanlinna interpolation problem for the case of an allowed Riemann surface $F$ may be formulated as follows. Let there be given a finite nonempty subset $A$ of $F$, maps $a \rightarrow \theta_{a}, a \rightarrow v(a), a \rightarrow p_{a}$, $a \in A$, where $\theta_{a}$ is a uniformizer satisfying $\theta_{a}(0)=a, v(a)$ is a nonnegative integer, and $p_{a}$ is a polynomial of degree not exceeding $v(a)$. The Pick-Nevanlinna interpolation problem for $F$ with the assigned data is to determine necessary and sufficient conditions in terms of the data for the existence of analytic functions $f$ on $F$ taking values of modulus at most one which satisfy the conditions 


$$
f \circ \theta_{a}(z)-p_{a}(z)=O\left(|z|^{\nu(a)+1}\right), \quad a \in A
$$

and to obtain information concerning functions satisfying the imposed conditions. If there is exactly one solution, the problem is said to be determinate.

We assert that if the problem is determinate, then the function $f$ in question is either a constant of modulus one or else is a map of constant finite valence of $F$ onto the open unit disk, and the value of the valence does not exceed

$$
\chi+\sum_{a \in A}[v(a)+1] .
$$

To see this, we proceed as follows. We put aside the trivial case where $f$ is a constant of modulus one and note that $\sum_{a \in A}[v(a)+1]>1$ and $\left|p_{a}(0)\right|<1, a \in A$. We fix $a_{0} \in A$ and introduce a Möbius transformation $\beta$ mapping $\{|z|<1\}$ onto $\{\operatorname{Re} z>0\}$ and satisfying $\beta\left[p_{a_{0}}(0)\right]=1$. With $a_{0}$ taking over the role of $a$ of the earlier sections and $A$ taking over the role of $B$, we take $A$ as in $\S 2$, the $v(a)$ now taking over the role of the $n(b)$. Then $\Lambda(\operatorname{Re} \beta \circ f)$ is a point of determinacy of $\Lambda(P)$ whose first $\chi+1$ components are zero. For if $u \in P$ satisfies $\Lambda(u)=$ $\Lambda(\operatorname{Re} \beta \circ f)$, then $u$ is the real part of an analytic function $g, g\left(\alpha_{0}\right)=1$, such that $\operatorname{inv} \beta \circ g=f$ by the determinacy of the stated problem. Hence $u=\operatorname{Re} \beta \circ f$, i.e., $\Lambda(\operatorname{Re} \beta \circ f)$ is a point of determinacy of $\Lambda(P)$. Consequently, $\operatorname{Re} \beta \circ f$ is a finite sum of minimal positive harmonic functions on $F$, where the number of summands may be taken as no greater than the bound given by (5.4) as we see with the aid of Theorem 4.2. It follows that $\beta \circ f$ is a map of $F$ onto $\{\operatorname{Re} z>0\}$ of constant valence whose value does not exceed the bound of (5.4). The function $f$ is a map of $F$ onto $\{|z|<1\}$ of constant valence whose value is the same as that for $\beta \circ f$, and hence not greater than the bound of (5.4) as asserted.

The extended Garabedian bound pertains to the situation where more than one admitted interpolating function exists. We suppose that this is the case, we fix $b \in F-A$, and let $W(b)$ denote the set of values $f(b)$, where $f$ is an admitted interpolating function. The set $W(b)$ is termed the Wertevorrat at $b$ for the given problem. It is compact and convex. An admitted interpolating function $f$ is termed extremal relative to $b$ (in the sense of $\mathrm{R}$. Nevanlinna [10]) provided that $f(b) \in \mathrm{fr} W(b)$. It was shown by Garabedian [5] for the plane case described above and by the author [6] for the case of an allowed Riemann surface $F$ that int $W(b) \neq \varnothing$ and that the augmented Pick-Nevanlinna problem which requires that $f$ satisfy in addition to the conditions of the stated problem the requirement that $f(b)=c$ where $c$ is a given point of $f r W(b)$ is determinate. We shall see that these facts may be concluded by the methods of the present paper. 
It will then follow that the Garabedian bound is a consequence of the bound (5.4) when one notes that augmentation by the condition at $b$ increases the sum $\sum[v(a)+1]$ by 1 .

We introduce $\beta$ and $\Lambda$ of the second preceding paragraph in the present context and, in addition, the augmentation $\Lambda_{1}$ of $\Lambda$ obtained by adjoining $\operatorname{Re} l(u ; b, 0)$ and $\operatorname{Im} l(u ; b, 0)$. The common element $x=$ $\Lambda(\operatorname{Re} \beta \circ f)$, where $f$ is a solution of the original problem (5.3) is not a point of determinacy of $\Lambda(P)$. By Corollary 5.2, int $V(x) \neq \varnothing$. Since $W(b)=\beta^{-1}[V(x)+1]$, we have int $W(b) \neq \varnothing$. By Theorem 5.1 the condition $f(b)=c$ implies that $\Lambda_{1}(\operatorname{Re} \beta \circ f)$ is a point of determinacy of $\Lambda_{1}(P)$. Hence we conclude that the augmented Pick-Nevanlinna problem is determinate. The bound

$$
\chi+\left\{1+\sum_{a \in A}[v(a)+1]\right\}
$$

for the value of the valence of an extremal is now apparent. This is the extended Garabedian bound.

$A$ remark on the sharpness of the bounds of (4.26) and (5.4). We shall now see that the bound (5.4) cannot be improved for given $\chi$ and $v^{\prime}=\sum[v(a)+1]$ when $v \geqq 2$. Determinacy in the case where $v=1$ occurs only for constant functions and so this case need not be considered further. The asserted sharpness of (5.4) implies the sharpness of (4.26) for given $\chi$ and $\sum[n(b)+1]$ when $\sum[n(b)+1] \geqq 2$. The case where $\sum[n(b)+1]=1$ will be treated separately.

To establish the asserted sharpness of (5.4) we consider a plane region $\Omega$ of connectivity $\chi+2$, each boundary component of which is a regular analytic closed Jordan curve. We fix $a, b(\neq a) \in \Omega$ and introduce a point $c \in \Omega$ distinct from $a$ and $b$ which will be allowed to tend to fr $\Omega$. We consider two extremal problems for functions analytic on $\Omega$ and taking values of modulus less than one. I. We impose the condition that $f$ have a zero at $a$ and seek $f$ maximizing $|f(b)|$. (Extremal problem of Ahlfors [1].) Let $f_{1}$ denote the solution normalized to satisfy $f_{1}(b)>0$. It is classical ([1], [5]) that $f_{1}$ has constant valence on the open unit disk, the value being $2+\chi$ (as is seen readily from (5.5) and the fact that an analytic function mapping $\Omega$ onto the open unit disk of constant valence is such that the value of the valence is at least $2+\chi)$. The case $v=2$ is thus cared for and we put it aside. II. We impose the condition that $f$ have a zero at $a$ and a zero of at least multiplicity $v-2$ at $c$ and seek $f$ maximizing $|f(b)|$. We let $f_{2}$ denote the extremal normalized to satisfy $f_{2}(b)>0$.

Now as $c$ tends to fr $\Omega$, the corresponding $f_{2}$ tends to $f_{1}$, uniformly on compact subsets of $\Omega$. Granting this assertion for the moment, we see 
that $f_{2}$ has $2+\chi$ zeros counted by multiplicity near the zeros of $f_{1}$ (by Hurwitz's theorem) and at least $\nu-2$ more at $c$ counted by multiplicity for $c \in \Omega-K$ where $K$ is a suitably chosen compact subset of $\Omega$. Hence using (5.4) we see that for such $c$ the valence of $f$ has the constant value on the open unit disk $\chi+v$, which is the Garabedian bound. We see that the bound (5.4) is sharp for assigned $v(\geqq 2)$ and $\chi$.

To treat the question of the convergence of $f_{2}$ to $f_{1}$ we proceed as follows. We note that there exists a function $\varphi_{c}$ analytic on $\Omega$, taking values of modulus less than one, having a zero of multiplicity $v-2$ at $c$ and no others, and such that $\varphi_{c}(b)>0$ and $\varphi_{c}$ tends to 1 uniformly on compact subsets of $\Omega$. We take $\varphi_{c}$ specified by the conditions $\varphi_{c}(b)>0$ and

$$
-\log \left|\varphi_{c}\right|=(v-2) G_{c}+\sum_{k=1}^{x+1}\left(t_{k} \omega_{k}+\left|t_{k}\right|\right)
$$

where $G_{c}$ is Green's function for $\Omega$ with pole at $c$, the $\omega_{k}$ are the harmonic measures of $\chi+1$ of the boundary components and the $t_{k}$ are fixed by the requirement that the periods of the conjugate of the right side of (5.6) are zero along $\chi+1$ fixed closed curves in $\Omega$ whose homology classes form a basis for the 1-dimensional homology group of $\Omega$. The point $c$ is taken as not lying on these curves. As $c$ tends to $\mathrm{fr} \Omega$, the $t_{k}$ tend to zero and we conclude that $\varphi_{c}$ tends to 1 , uniformly on compact subsets of $\Omega$. Now

$$
f_{1}(b) \varphi_{c}(b) \leqq f_{2}(b) \leqq f_{1}(b) .
$$

Hence $f_{2}$ converges to $f_{1}$ as asserted.

There remains to be considered the sharpness of (4.26) when $\sum[n(b)+1]=1$. The case where $\chi=-1$ is to be put aside, the question of determinacy being meaningless. When $\chi \geqq 0$, we refer again to $\Omega$ and $a \in \Omega$ as introduced. Here it is convenient to take the $\gamma_{k}$ as $\chi+1$ components of the level set $\left\{G_{a}=\lambda\right\}$ where $\lambda$ is small and positive. We recall that the origin of $R^{x+1}$ is an inner point of $\Lambda(P)$ taken relative to the present situation. We consider the two points on fr $\Lambda(P)$ whose first $\chi$ components are zero. The preimage with respect to $\Lambda \mid P$ of at least one of these points is the sum of $\chi+1$ minimal positive harmonic functions on $\Omega$ which have their singularities on distinct components of fr $\Omega$. It is to be observed that the preimages with respect to $\Lambda \mid P$ of both of the points of fr $\Lambda(P)$ in question have singularities on the components of fr $\Omega$ close to $\gamma_{1}, \cdots, \gamma_{\chi}$. (Otherwise the first $\chi$ periods would not all be zero.) If neither preimage had a singularity on the component of fr $\Omega$ near $\gamma_{\chi+1}$, the $(\chi+1)$ st components of the points of $\operatorname{fr} \Lambda(P)$ under consideration would have the same sign. This is not possible since the origin 
of $R^{x+1}$ is an inner point of $\Lambda(P)$. We conclude that the bound $\chi+1$ prevailing in this case cannot be improved. The obvious conventions are to be understood when $\chi=0$.

(c) An extremal problem of S. Fisher. The following problem has been treated by $\mathrm{S}$. Fisher [4]:

Let $a$ and $b$ be distinct points of an admitted Riemann surface $F$. Let $B$ denote the family of functions $f$, analytic on $F$, taking values of modulus less than one, and satisfying both $f(a)=0$ and $f(p) \neq 0, p \neq a$. Determine the nature of the $f$ maximizing $|f(b)|$.

Essentially, the result of Fisher is: An extremal function $f$ for this problem has the property that

$$
-\log |f|-n(a ; f) G_{a}
$$

may be represented as the sum of at most $\chi+1$ minimal positive harmonic functions on $F, n(a ; f)$ being the multiplicity of $f$ at $a$. Further the situation where there is more than one normalized extremal function does indeed occur [4], p. 1188, normalization being understood in the sense that the function in question takes a positive value at $b$.

We shall now see that the set of normalized extremals is finite. The case where $F$ is simply-connected is immediate and will be put aside. We shall approach the question by introducing a countable family of auxiliary extremal problems defined on appropriate subfamilies of $B$, which are such that each has a unique normalized solution and that a normalized extremal of the problem of Fisher is also an extremal for one of these problems. It will be seen that only a finite number of the normalized extremals for the auxiliary problems may compete as candidates to be a normalized extremal of the problem of Fisher. The asserted finiteness property follows.

We now turn to the details. For convenience we let $b$ take over the role of $a$ of $\S 2$ and suppose to put aside unessential questions that the curves $\gamma_{1}, \cdots, \gamma_{x+1}$ do not contain $a$. The functions $-\log |f|, f \in B$, are characterized by the fact that they admit a representation of the form

$$
n G_{a}+u
$$

where $n$ is a positive integer, $u$ is a nonnegative harmonic function on $F$, and $\omega\left(n G_{a}+u ; \gamma_{j}\right) /(2 \pi)$ is an integer, $j=1, \cdots, \chi+1$. Given $\left(n, v_{1}, \cdots, v_{\chi+1}\right)$ where $n$ is a positive integer and the $v_{j}$ are integers, we introduce the family $H\left(n, v_{1}, \cdots, v_{x+1}\right)$ of non-negative harmonic functions $u$ on $F$ satisfying

$$
\omega\left(n G_{a}+u ; \gamma_{j}\right)=2 \pi v_{j}, \quad j=1, \cdots, \chi+1 .
$$

It is clear that $f \in B$ has multiplicity $n$ at $a$ and satisfies

$$
\omega\left(-\log |f| ; \gamma_{j}\right)=2 \pi v_{j}, \quad j=1, \cdots, \chi+1,
$$


if and only if $-\log |f|$ admits a representation of the form (5.9) with $u \in H\left(n, v_{1}, \cdots, v_{x+1}\right)$. Given $\left(n, v_{1}, \cdots, v_{x+1}\right), f_{0}$ maximizes $|f(b)|$ in the family of $f \in B$ which have multiplicity $n$ at $a$ and satisfy (5.11) if and only if the corresponding $u_{0}=-\log \left|f_{0}\right|-n G_{a}$ minimizes $u(b)$ in the family $H\left(n, v_{1}, \cdots, v_{\chi+1}\right)$.

There are two cases to consider: (1) $\omega\left(n G_{a} ; \gamma_{j}\right)=2 \pi v_{j}, \quad j=$ $1, \cdots, \chi+1 ;(2)$ the assumption of $(1)$ is not fulfilled.

In case (1), trivially, the minimizing $u$ is the constant 0 . This case is indeed realizable as one sees on considering a two-sheeted covering of the open unit disk with simple ramification points over 0 and some other point of the open unit disk and no others, the point $a$ being the ramification point over zero and the point $b$ being the other.

We turn to case (2). Here a minimizing $u$ is positive. We introduce $\Lambda$ formed from the period system $\omega\left(; \gamma_{j}\right), j=1, \cdots, \chi+1$, as in $\S 2$ and show that $u \in H\left(n, v_{1}, \cdots, v_{\chi+1}\right)$ minimizes the value at $b$ if and only if $\Lambda[u / u(b)] \in$ fr $\Lambda(P)$. Indeed, let $w$ be the unique member of $P$ such that $\Lambda(w) \in \operatorname{fr} \Lambda(P)$ and $\Lambda[u / u(b)]$ is a positive multiple $\varrho$ (necessarily $\leqq 1$ ) of $\Lambda(w)$. Then $u$ minimizes the value at $b$ if and only if $\varrho=1$. To see this we proceed contrapositively. If $\varrho<1$, then $\Lambda(u)=$ $\Lambda[\varrho u(b) w]$ and so $u(b)$ is not the least value at $b$ of the members of $H\left(n, v_{1}, \cdots, v_{\chi+1}\right)$. If $u \in H\left(n, v_{1}, \cdots, v_{x+1}\right)$ does not minimize the value at $b$, let $v \in H\left(n, v_{1}, \cdots, v_{\chi+1}\right)$ satisfy $v(b)<u(b)$. From

$$
\Lambda\left[\frac{v}{v(b)}\right]=\frac{u(b) \varrho}{v(b)} \Lambda(w),
$$

we conclude that $u(b) \varrho / v(b) \leqq 1$ and hence that $\varrho<1$.

It now follows that in case (2) there is a unique minimizing $u$ and that it admits a representation as the sum of at most $\chi+1$ minimal positive harmonic functions on $F$. To the auxiliary extremal problem associated with an assigned multiplicity at $a$ and the period conditions (5.11) there therefore corresponds a unique normalized extremal.

Since

$$
-\log |f(b)| \geqq n(a ; f) G_{a}(b), \quad f \in B,
$$

it is clear that the extremals of the auxiliary problems enter into consideration only for a finite set of $n$. For given $n$ only a finite set of $\left(v_{1}, \cdots, v_{\chi+1}\right)$ need be considered, for the minimum value at $b$ of members of $H\left(n, v_{1}, \cdots, v_{\chi+1}\right)$ is at least as large as

$$
\left[2 \pi\left(\sum v_{j}^{2}\right)^{1 / 2}-A\right] / B,
$$

where $A$ does not depend on $\left(v_{1}, \cdots, v_{\chi+1}\right)$ and $B$ is the maximum of the euclidean lengths of the vectors $x \in \operatorname{fr} \Lambda(P)$. 
We conclude that there are only a finite number of normalized extremals in $B$ for the original problem.

In the cited paper of S. Fisher analogous problems are considered in which several zeros or poles are allowed. On considering the decomposition of the logarithm of the modulus with the aid of Green's functions, we find that the above developments are applicable.

6. Support functions. We recall that the support function $M$ of a nonempty compact convex set $K \subset R^{m}$ is defined as the function with domain $R^{m}$ given by

$$
M(y)=\max _{x \in K}<x, y>,
$$

$<$, > denoting the inner product for $R^{m}$. The function $M$ is subadditive and homogeneous of order one relative to nonnegative scalar factors. It is a classical consequence of this fact that $M$ is continuous [2].

When $K=\Lambda(P), M$ is determined by $\Phi$ since the points of $\Lambda(P)$ are given barycentrically in terms of $\Phi$. In fact,

$$
M(y)=\max _{q \in \Gamma}<\Phi(q), y>.
$$

Since the origin $O$ of $R^{m}$ is an inner point of $\Lambda(P), M(y)>0$ for $y \neq 0$. From standard results concerning convex sets [2] we have

$$
\Lambda(P)=\bigcap_{y \in R^{m}}\{x:\langle x, y\rangle \leqq M(y)\} .
$$

On noting that a supporting plane of $\Lambda(P)$ passes through each point of fr $\Lambda(P)$ we see that $x\left(\in R^{m}\right)$ belongs to $\Lambda(P)$ if and only if

$$
\mu(x)=\min _{\langle y, y\rangle=1}[M(y)-\langle x, y\rangle] \geqq 0,
$$

and that $x$ is a point of determinacy of $\Lambda(P)$ if and only if $\mu(x)=0$.

We now suppose that $m>\chi+1$ and let $n=m-(\chi+1)$ and let $\pi$ denote the projection map of $R^{m}$ onto $R^{n}$ defined by

$$
\pi\left(x_{1}, \cdots, x_{m}\right)=\left(x_{\chi+2}, \cdots, x_{m}\right) .
$$

Let $P_{0}$ denote the set of $u \in P$ satisfying $\omega\left(u ; \gamma_{k}\right)=0, k=$ $1, \cdots, \chi+1$. The set $\pi\left[\Lambda\left(P_{0}\right)\right]$ is compact and convex. We seek to determine its support function in terms of $M$ and hence ultimately in terms of $\Phi$. The significance of $\pi\left[\Lambda\left(P_{0}\right)\right]$ lies in the fact that the points of $\pi\left[\Lambda\left(P_{0}\right)\right]$ correspond to the data of Pick-Nevanlinna interpolation problems with the harmless modification that one considers analytic functions with positive real part subject to the normalization that they take the value 1 at $a$ rather than analytic functions taking values of 
modulus less than one (the trivial case involving constants of modulus one being set aside). By the extension of Theorem 5.1 indicated in the remark following Corollary 5.2 we see that $x \in \Lambda\left(P_{0}\right)$ is a point of determinacy of $\Lambda\left(P_{0}\right)$ if and only if $\pi(x) \in \mathrm{fr} \pi\left[\Lambda\left(P_{0}\right)\right]$. [The obvious meaning of determinacy that there is exactly one element of $u \in P_{0}$ such that $\Lambda(u)=x$ is understood.]

To obtain the support function of $\pi\left[\Lambda\left(P_{0}\right)\right]$ in terms of $M$ we proceed as follows. Given $y \in R^{n}$, we let $l(y)$ denote the unique preimage of $y$ with respect to $\pi$ whose first $\chi+1$ coordinates are zero and introduce

$$
M_{0}(y)=\min _{w} M[l(y)+w],
$$

where $w \in R^{m}$ and its last $n$ coordinates are zero. It is readily verified that the minimum in question is attained and that $M_{0}$ is subadditive and homogeneous (of order 1 ) with respect to nonnegative scalar factors. We shall show

Theorem 6.1. $M_{0}$ is the support function of $\pi\left[\Lambda\left(P_{0}\right)\right]$.

Proof. We put aside the obvious case where $\chi=-1$. We observe that $x \in \pi\left[\Lambda\left(P_{0}\right)\right]$ if and only if

$$
<x, y>_{n} \leqq M[l(y)+w]
$$

for all allowed $y$ and $w,<,>_{n}$ signifying the standard inner product of $R^{n}$. It follows that $x \in \pi\left[\Lambda\left(P_{0}\right)\right]$ if and only if

$$
<x, y>_{n} \leqq M_{0}(y), \quad y \in R^{n} .
$$

Further $x \in \operatorname{fr} \pi\left[\Lambda\left(P_{0}\right)\right]$ if and only if

$$
\min _{\langle y, y\rangle_{n}=1}\left[M_{0}(y)-<x, y>_{n}\right]=0 .
$$

To establish this assertion we proceed as follows. It is to be noted that $M$ vanishes only at the origin $O$ of $R^{m}$. When $x \in \operatorname{fr} \pi\left[\Lambda\left(P_{0}\right)\right]$, equality in (6.7) holds for some $y$ and $w$ with $l(y)+w \neq 0$, as follows from the indicated extension of Theorem 5.1. If $l(y)=0$, then $M(w)=$ $\langle x, y\rangle_{n}=0$ and so $w=0$ and consequently $l(y)+w=0$. This is not possible. We conclude (6.9). If (6.9) holds, then at least $x \in \pi\left[\Lambda\left(P_{0}\right)\right]$ and equality holds in (6.7) for some $y$ not the origin of $R^{n}$ and some allowed $w$. Hence $l(x) \in \operatorname{fr} \Lambda(P)$. A second application of the indicated extension of Theorem 5.1 shows that $x \in \operatorname{fr} \pi\left[\Lambda\left(P_{0}\right)\right]$.

We now verify that $M_{0}$ is the support function of $\pi\left[\Lambda\left(P_{0}\right)\right]$. Because of the noted subadditivity and homogeneity of $M_{0}$, by a classical result of the theory of convex sets [2, p. 26], [11], $M_{0}$ is the support function of a nonempty compact convex set. By the representation of a compact convex set in terms of its support function, $M_{0}$ is the support function of 


$$
\bigcap_{y \in R^{n}}\left\{\langle x, y\rangle \leqq M_{0}(y)\right\} .
$$

It follows on reference to (6.8) that $M_{0}$ is the support function of $\pi\left[\Lambda\left(P_{0}\right)\right]$.

We remark that $M_{0}$ vanishes only at the origin of $R^{n}$, as we see on noting that the origin of $R^{n}$ is an inner point of $\pi\left[\Lambda\left(P_{0}\right)\right]$.

7. The Schwarz Lemma extremal problem. The Schwarz Lemma extremal problem for a Riemann surface $F$ may be solved in terms of $\Phi$ and $M$ as we shall now see. We recall that one is concerned with given points $a, b(\neq a) \in F$ and one seeks to maximize $|f(b)|$ in the family of functions $f$ analytic on $F$, taking values of modulus less than one, and satisfying $f(a)=0$. The set of values $f(b)$ is a closed circular disk with center 0 having a positive radius less than one. With the aid of the Möbius transformation $z \rightarrow(1+z) /(1-z)$ the problem reduces to maximizing $\operatorname{Re} g(b)$ in the family of functions $g$ analytic on $F$, having positive real parts and normalized to take the value 1 at $a$. With $\Lambda(u)$ now having as components the $\omega\left(u ; \gamma_{k}\right), \operatorname{Re} l(u ; b, 0)$ and $\operatorname{Im} l(u ; b, 0)$ and with $\operatorname{Re} l(u ; b, 0)$ the $(\chi+2)$ nd component of $\Lambda(u)$ we see that the maximum $\operatorname{Re} g(b)$ is $1+M_{0}(1,0)$. It follows that the maximum $|f(b)|$ is

$$
M_{0}(1,0) /\left[M_{0}(1,0)+2\right] \text {. }
$$

The extremal $g$ may be determined as follows. Let $y=\left(y_{1}, \cdots, y_{x+1}, 1,0\right)$ be such that $M(y)=M_{0}(1,0)$ and let $E$ denote the set of $q \in \Gamma$ satisfying $\langle\Phi(q), y\rangle=M(y)$. Then the $\Phi(q), q \in \mathbb{E}$, are linearly independent. The point $\left(0, \cdots, 0, M_{0}(1,0), 0\right)$ admits a unique barycentric representation of the form $\sum_{q \in E} t(q) \Phi(q), t(q) \geqq 0, \sum t(q)=1$. The $t(q)$ are determined algebraically on the basis of these facts. For the extremal $g$ we have $\operatorname{Re} g=\sum t(q) u_{q}$. The extremal $g$ and hence the extremal $f$ normalized to take a positive value at $b$ are thereby determined.

8. The Painlevé problem for Riemann surfaces. The support function $M_{0}$ leads to a theoretical solution of the Painlevé problem for Riemann surfaces. A solution for the case of plane regions was given by P. R. Garabedian [5]. The problem of Painlevé, we recall, is to obtain a necessary and sufficient condition for a given Riemann surface to admit non-constant bounded analytic functions.

We suppose that we have at hand a given noncompact Riemann surface $\Omega$ and that $\left(\Omega_{n}\right)_{1}^{\infty}$ is an exhaustion of $\Omega$ satisfying the following conditions: $\Omega_{n}$ is relatively compact, $\bar{\Omega}_{n} \subset \Omega_{n+1}$, fr $\Omega_{n}=\operatorname{fr}\left(\Omega-\bar{\Omega}_{n}\right)$ and consists of a finite number of disjoint regular analytic Jordan curves, $n=1,2, \cdots$. We fix $a \in \Omega_{1}$ and a local uniformizer $\theta, \theta(0)=a$. Given a positive integer $v$ we introduce $\Lambda_{n, v}$ on the space of harmonic 
functions on $\Omega_{n}$ as a map $\Lambda$ relative to $\Omega_{n}$ formed in terms of the periods of the conjugates on curves $\gamma_{1}, \cdots, \gamma_{x_{n}+1}$, as above and the real and imaginary parts of

$$
\frac{2}{k !}(u \circ \theta)_{z}^{(k-1)}(0), \quad k=1, \cdots, v .
$$

Here $\chi_{n}$ denotes the Euler characteristic of $\bar{\Omega}_{n}$. It is supposed that the components of $\Lambda_{n, v}(u)$ are so arranged that the last component is

$$
\frac{2}{\nu !} \operatorname{Im}(u \circ \theta)_{z}^{(\nu-1)}(0) \text {. }
$$

Let $M_{0}$ of $(6.6)$ be taken relative to $\Lambda_{n, v}$ and let $p(n, v)$ denote the value that $M_{0}$ takes at the coordinate vector of $R^{2 v}$ whose last component is 1 and others are 0 . It is to be observed that $n \rightarrow p(n, v)$ is decreasing, $v=1,2, \cdots$.

We shall show that the double sequence $(p(n, v))$ yields a necessary and sufficient condition of the desired kind. In fact, we have

The or e m 8.1. A necessary and sufficient condition for $\Omega$ to admit nonconstant bounded analytic functions is that

$$
\sup _{\nu}\left[\lim _{n \rightarrow \infty} p(n, v)\right]>0 .
$$

Proof. Suppose that $\Omega$ admits a nonconstant bounded analytic function. We are then assured that there exists a function $b$ analytic on $\Omega$, nonconstant, taking values of modulus less than one and satisfying $b(a)=0$. For some $\eta \in\{1,-1, i,-i\}$, and $v$, the function $f=$ $1+\eta b$, which is analytic on $\Omega$ and nonconstant, and has positive real part, satisfies

$$
\operatorname{Im}(f \circ \theta)^{(\nu)}(0)>0 .
$$

For this $v$ and all positive integers $n$, the inequality

$$
p(n, v) \geqq \operatorname{Im}(f \circ \theta)^{(v)}(0) / v !
$$

holds since $\operatorname{Re}\left(f \mid \Omega_{n}\right)$ belongs to $P_{0}$ relative to $\Omega_{n}$. The condition (8.3) follows.

Suppose now that the condition (8.3) holds. For some $v$ we have $\lim _{n \rightarrow \infty} p(n, v)>0$. It follows that there exists a sequence $\left(f_{n}\right)$, where $f_{n}$ is analytic and has positive real part on $\Omega_{n}, f_{n}(a)=1$, and

$$
\inf _{n} \operatorname{Im}\left(f_{n} \circ \theta\right)^{(\nu)}(0)>0 .
$$

It suffices to take $f_{n}$ as an extremal function in the class of functions $f$ analytic on $\Omega_{n}$, having positive real part and satisfying $f(a)=1$, which 
maximizes $\operatorname{Im}(f \circ \theta)^{(\nu)}(0)$. On taking a subsequence of $\left(f_{n}\right)$ which converges uniformly on compact subsets of $\Omega$, we see that its limit is a nonconstant analytic function on $\Omega$ with positive real part. We conclude that $\Omega$ admits nonconstant bounded analytic functions.

The result that we have obtained may be restated in terms of the classification theory of Riemann surfaces. We recall that $O_{A B}$ denotes the class of Riemann surfaces which do not admit nonconstant bounded analytic functions. Theorem 8.1 may be recast to read: $\Omega \in O_{A B}$ if and only if $\lim _{n \rightarrow \infty} p(n, v)=0, v=1,2, \cdots$.

One matter calls for comment. Given a positive integer $v_{0}$ we shall construct with the aid of a method due to P. J. Myrberg [8] an $\Omega \notin O_{A B}$ which contains a point $a$ such that every bounded analytic function $f$ on $\Omega$ has the property that $(f \circ \theta)^{(\nu)}(0)=0, v=1, \cdots, v_{0}$. Here we have $\lim _{n \rightarrow \infty} p(n, v)=0, v=1, \cdots, v_{0}$. Hence with $E$ a nonempty, finite set of positive integers the condition

$$
\max _{v \in E}\left[\lim _{n \rightarrow \infty} p(n, v)\right]>0
$$

is not necessary and sufficient for $\Omega \notin O_{A B}$.

To construct an $\Omega$ of the desired kind, it suffices to consider the $\left(v_{0}+1\right)$-sheeted covering of the open disk defined by $z \rightarrow z^{\nu_{0}+1}$ and to introduce a sequence of slits on two "successive sheets" lying over the interval $(0,1)$ such that their endpoints tend to one and satisfy the Blaschke divergence condition. On joining the two successive sheets across the slits in the standard manner (the upper edge of one sheet being joined to the lower edge of the other) we obtain a $\left(v_{0}+1\right)$-sheeted covering of the open unit disk which has the property that a bounded analytic function $f$ on it takes the same value at all points of the surface lying over a given point of the open unit disk. It then follows on taking $a$ as the unique preimage of 0 with respect to the modified covering map that $(f \circ \theta)^{(v)}(0)=0, v=1, \cdots, v_{0}$.

9. Some allied problems. In this section we shall indicate how the methods of the present paper may be applied to the study of body problems for certain other classes of harmonic functions, for which, in fact, the results turn out to have a very simple character. We shall be concerned with the harmonic Hardy classes $h_{p}$ on $F, 1<p \leqq+\infty$. To be precise, by the class $h_{p}$ we understand the class of real-valued harmonic functions $u$ on $F$ satisfying the condition that $|u|^{p}$ possess a harmonic majorant when $1<p<+\infty$ and the condition that $u$ be bounded when $p=+\infty$. In the former case we introduce the norm 


$$
\|u\|_{p}=\left(\frac{i}{2 \pi} \int_{\Gamma}\left|u^{*}\right|^{p} \delta G_{a}\right)^{1 / p},
$$

where $u^{*}$ denotes the Fatou boundary function of $u$. In the latter case we define $\|u\|_{\infty}$ to be $\sup |u|$. [It is to be observed that we are putting aside the case where $p=1$ which does not fit into the developments of this section because of the breakdown of convenient properties. This case can be treated in terms of differences of nonnegative harmonic functions and may, in fact, be referred to the earlier developments of the present paper.]

We propose to characterize the image of the unit ball $B_{p}$ of $h_{p}$ with respect to a map $\Lambda_{1}$ obtained from $\Lambda$ by augmentation, the component

$$
u(a)=\frac{i}{2 \pi} \int_{\Gamma} u^{*} \delta G_{a}
$$

being added. We let $\Psi$ denote the corresponding augmentation of $\Phi$. It is obtained merely by the adjunction of a component equal to one. The role of $\Lambda_{1}$ for interpolation problems is apparent. It is easy to see that $\Lambda_{1}\left(B_{p}\right)$ is a compact convex set. We shall determine the support function of $\Lambda_{1}\left(B_{p}\right)$ in terms of $\Psi$ and thereby give a characterization of $\Lambda_{1}\left(B_{p}\right)$ in terms of $\Psi$.

We start with the observation that

$$
\Lambda_{1}(u)=\frac{i}{2 \pi} \int_{\Gamma} \Psi u^{*} \delta G_{a}, \quad u \in h_{p} .
$$

Hence for $y \in R^{m+1}$ we have

$$
\left\langle\Lambda_{1}(u), y>=\frac{i}{2 \pi} \int_{\Gamma}\left\langle\Psi, y>u^{*} \delta G_{a} .\right.\right.
$$

[Here $\langle\Psi, y\rangle$ denotes the function $q \rightarrow\langle\Psi(q), y\rangle, q \in \Gamma$.] We first consider the case where $p=+\infty$.

In this case using (9.4) we conclude that

$$
\left\langle\Lambda_{1}(u), y\right\rangle \leqq \frac{i}{2 \pi} \int_{\Gamma}|<\Psi, y>| \delta G_{a},
$$

and that when $y$ is not the zero vector, equality holds if and only if $u^{*}(q)=s g\langle\Psi(q), y\rangle$ save possibly at the zeros of $\langle\Psi, y\rangle$. It is to be noted that the component functions of $\Psi$ are linearly independent over $R$. It follows that $s_{\infty}$, the support function of $A_{1}\left(B_{\infty}\right)$, is given by

$$
s_{\infty}(y)=\frac{i}{2 \pi} \int_{\Gamma}|<\Psi, y\rangle \mid \delta G_{\alpha} .
$$


From the condition for equality in (9.5), it follows that there is a unique point in the supporting plane $\langle x, y\rangle=s_{\infty}(y)$ belonging to $\Lambda_{1}\left(B_{\infty}\right)$. Further, as follows from, say, the linear independence of the components of $\Psi$, the origin of $R^{m+1}$ is an interior point of $\Lambda_{1}\left(B_{\infty}\right)$.

It is easily verified that the points of determinacy of $A_{1}\left(B_{\infty}\right)$ (with respect to $\left.B_{\infty}\right)$ are exactly the frontier points of $\Lambda_{1}\left(B_{\infty}\right)$. To see this we proceed as follows. We recall that there is a supporting plane of $\Lambda_{1}\left(B_{\infty}\right)$ passing through a given point of $\operatorname{fr} \Lambda_{1}\left(B_{\infty}\right)$. Let $x \in \operatorname{fr} \Lambda_{1}\left(B_{\infty}\right)$ and let $y \neq 0$ be such that $\langle x, y\rangle=s_{\infty}(y), O$ denoting the origin of $R^{m+1}$. The facts concerning the case of equality in (9.5) show that $x$ is a point of determinacy. We note that $O$ is not a point of determinacy. Indeed, it suffices to introduce harmonic measures of $m+2$ disjoint subarcs of $\Gamma$ and to note that their images with respect to $\Lambda_{1}$ are linearly dependent over $R$ to be assured that $O$ is not a point of determinacy. It follows that a point of int $\Lambda_{1}\left(B_{\infty}\right)$ is not determinate. Indeed, such a point has a preimage $u$ with respect to $\Lambda_{1}$ satisfying $\sup |u|<1$, so that with $v$ a preimage of $O$ with respect to $\Lambda_{1}$ which is small but not the constant 0 we have $u+v \in B_{\infty}$ and $\Lambda_{1}(u+v)=\Lambda_{1}(u)$. Hence a point of determinacy is a point of $\operatorname{fr} A_{1}\left(B_{\infty}\right)$. The identity of $\mathrm{fr} \Lambda_{1}\left(B_{\infty}\right)$ and the set of points of determinacy of $A_{1}\left(B_{\infty}\right)$ with respect to $B_{\infty}$ follows.

For $y$ not the origin and with $u_{y}$ given by

$$
u_{y}(t)=\frac{i}{2 \pi} \int_{\Gamma} s g\langle\Psi, y\rangle \delta G_{t}, \quad t \in F,
$$

we see that $\Lambda_{1}\left(u_{y}\right)$ is the unique point of $\operatorname{fr} \Lambda_{1}\left(B_{\infty}\right)$ in the supporting plane $\left\{\langle x, y\rangle=s_{\infty}(y)\right\}$. It is now easily concluded that $y \rightarrow \Lambda_{1}\left(u_{y}\right)$ maps $\{\|y\|=1\}$ onto fr $\Lambda_{1}\left(B_{\infty}\right)$.

There remains to be considered the case where $1<p<+\infty$. Here the Hölder inequality and the standard facts concerning the situation of equality play an essential role. From (9.4) we obtain

$$
\left\langle\Lambda_{1}(u), y>\leqq\left[\frac{i}{2 \pi} \int_{\Gamma}|<\Psi, y>|^{p /(p-1)} \delta G_{a}\right]^{(p-1) / p}, \quad u \in B_{p},\right.
$$

and the fact when $y$ is not the zero vector, equality occurs in (9.8) if and only if

$$
u^{*}(q)=c(y)|<\Psi(q), y>|^{1 /(p-1)} s g<\Psi(q), y>, \quad q \in \Gamma,
$$

where $c(y)$ is the positive number yielding a function of $p$-norm 1 , namely, $\|<\Psi, y>\|_{q}^{-1 /(p-1)}$. The details required to complete the discussion of the case at hand are straightforward. We find that the support function, $s_{p}$, for $\Lambda_{1}\left(B_{p}\right)$ is given by 


$$
s_{p}(y)=\left[\left.\frac{i}{2 \pi} \int_{\Gamma}|<\Psi, y\rangle\right|^{p /(p-1)} \delta G_{a}\right]^{(p-1) / p},
$$

the determinacy set relative to $B_{p}$ is $\operatorname{fr} A_{1}\left(B_{p}\right)$, and that with $y$ given by

$$
u_{y}(t)=\frac{i}{2 \pi} \int_{\Gamma} c(y)|<\Psi, y>|^{1 /(p-1)} s g<\Psi, y>\delta G_{t}, \quad t \in F,
$$

$y \rightarrow \Lambda_{1}\left(u_{y}\right)$ maps $\{\|y\|=1\}$ univalently onto fr $\Lambda_{1}\left(B_{p}\right)$. Only the question of univalence calls for comment. If $\Lambda_{1}\left(u_{y}\right)=\Lambda_{1}\left(u_{z}\right)$ where $\|y\|=\|z\|=1$, then $u_{y}=u_{z}$. Hence $\langle\Psi, y\rangle=c\langle\Psi, z\rangle$ where $c$ is a positive number. By the linear independence of the components of $\Psi$, $y=c z$. Since $\|y\|=\|z\|=1$, we conclude that $c=1$. The asserted univalence follows.

Univalence does not hold for the corresponding map in the $h_{\infty}$ theory. To see this it suffices to consider the classical Carathéodory setting: $F=$ $\{|z|<1\}, l(u ; 0, k), k=1, \cdots, n(>1), u(0)$, and to note that $|z-1|^{2 m},|z|=1, m=1, \cdots, n$, yield $\langle\Psi, y\rangle$ for which $s g\langle\Psi, y\rangle$ are equal but the $y$ are not proportional.

The support functions which have been determined in this section may be applied to study "Painlevé" problems relative to the classes $h_{p}$ $(1<p \leqq+\infty)$ and $H_{p}(1<p<+\infty)$ on unrestricted noncompact Riemann surfaces. We plan to return to these questions and allied extremal problems in a subsequent paper.

It is to be remarked that Nehari [9] has studied extremal problems for bounded analytic functions on multiply-connected plane regions of finite connectivity by referring the question to the study of harmonic functions taking values in $[0,1]$. The relationship of the work of Nehari to the indicated study of $A_{1}\left(B_{\infty}\right)$ is apparent. His results which are based on variational methods have a qualitative character.

\section{References}

[1] AhlFORS, L. V.: Open Riemann surfaces and extremal problems on compact subregions. - Comment. Math. Helv. 24, 1950, 100-134.

[2] BonNESEN, T., and W. FENCHEL: Theorie der konvexen Körper. - Ergebnisse der Mathematik und ihrer Grenzgebiete 3:1, Verlag von Julius Springer, Berlin, 1934.

[3] CARATHÉODORY, C.: Über den Variabilitätsbereich der Fourier'schen Konstanten von positiven harmonischen Funktionen. - Rend. Circ. Mat. Palermo 32, 1911, 193-217.

[4] Fisher, S.: Non-linear extremal problems in $H^{\infty}$. - Indiana Univ. Math. J. $22,1972 / 1973,1183-1190$. 
[5] Garabedian, P. R.: Schwarz's lemma and the Szegö kernel function. - Trans. Amer. Math. Soc. 67, 1949, 1-35.

[6] HEIns, M.: Hardy classes on Riemann surfaces. - Lecture Notes in Mathematics 98, Springer-Verlag, Berlin-Heidelberg-New York, 1969.

[7] -»- Nonpersistence of the Grenzkreis phenomenon for Pick-Nevanlinna interpolation on annuli. - Ann. Acad. Sci. Fenn. Ser. A I 596, 1975, 1-21.

[8] MyrberG, P. J.: Über die analytische Fortsetzung von beschränkten Funktionen. - Ann. Acad. Sci. Fenn. Ser. A I 58, 1949, 1-7.

[9] NEHARI, Z.: Extremal problems in the theory of bounded analytic functions. Amer. J. Math. 73, 1951, 78-106.

[10] Nevanlinna, R.: Über beschränkte analytische Funktionen. - Commentationes in honorem Ernesti Leonardi Lindelöf. Ann. Acad. Sci. Fenn. Ser. A. $32: 7,1929,1-75$.

[11] RADEMAChER, H.: Zur Theorie der Minkowskischen Stützebenenfunktion. S.-B. Berlin Math. Ges. 20, 1921, 14-19.

[12] READ, A.: A converse of Cauchy's theorem and applications to extremal problems. - Acta Math. 100, 1958, 1-22.

[13] RIEsz, F.: Über Potenzreihen mit vorgeschriebenen Anfangsgliedern. - Acta Math. 42, 1920, 145-171.

[14] Seminar on convex sets. - [Mimeographed notes.] Institute for Advanced Study, Princeton, New Jersey, 1949/1950.

University of Maryland

Department of Mathematics

College Park, Maryland 20742

USA

Received 1 April 1975 